\section{Ecists}

${ }^{1}$ Department of Respiratory Medicine, St James's University Hospital, Leeds, UK

${ }^{2}$ Department of Cardiovascular and Respiratory Studies, Castle Hill Hospital, Hull, UK

\section{Correspondence to} Dr Georgina Esterbrook, Department of Respiratory Medicine, St James's University Hospital, Beckett Street, Leeds LS9 7TF, UK; Georginaesterbrook@hotmail. com

Received 30 July 2012 Accepted 9 August 2012 Published Online First 12 September 2012

To cite: Esterbrook $\mathrm{G}$, Molyneux I, Clark AL, et al. Thorax 2013;68:302-304.

CASE BASED DISCUSSION

\title{
A case of cough and breathlessness
}

\author{
Georgina Esterbrook, ${ }^{1}$ Ian Molyneux, ${ }^{2}$ Andrew L Clark, ${ }^{2}$ Alyn H Morice ${ }^{2}$
}

GE: A 64-year-old man never smoker with a history of dyspnoea and cough was referred for a respiratory opinion. The cough was sporadic but progressive over a 9 month period. It was associated with unpredictable, brief nocturnal paroxysms of breathlessness. There were also coughing bouts associated with phonation and with meals but no sputum production, fever or weight loss. There was no significant past medical history and he was not taking any medication. Physical examination was unremarkable.

IM: This gentleman presents with a chronic, non-productive cough and episodic breathlessness. The duration of his symptoms makes an infective cause unlikely and in the absence of a smoking history, the most likely diagnosis is an asthmatic cough. However, in this age group, a de novo presentation of asthma is unusual and other common causes of chronic cough such as gastro-oesophageal reflux should be considered. There are no specific pointers to a rarer diagnosis or to lung cancer; however, the latter must be actively excluded. Cough and dyspnoea are common manifestations of bronchiecetasis, interstitial lung disease and left ventricular failure but one would expect other associated features. Further investigations are required to clarify the differential diagnosis.

GE: Chest radiograph was normal with no cardiomegaly and normal lung fields. Spirometry was normal (forced expiratory volume in the first second 2.9L, 95\% predicted; forced vital capacity 3.6L, 91\%; maximal expiratory flow 50 130\%). There was poor R wave progression on ECG and an echocardiogram showed left ventricular hypertrophy with an estimated pulmonary artery systolic pressure of $44 \mathrm{~mm} \mathrm{Hg}+$ right atrial pressure. Left ventricular systolic function was reported as good. He achieved $525 \mathrm{~m}$ on a 6 -min walk with no desaturation on oximetry but he experienced significant breathlessness. A CT scan of the thorax demonstrated normal lungs with no evidence of interstitial or small airways disease and no pulmonary emboli. He scored 50/70 on the Hull Airway Reflux Questionnaire (HARQ). ${ }^{1}$ An airway $\mathrm{pH}$ study and barium swallow confirmed the presence of reflux.

AHM: The cardiac investigations are abnormal, but the reported good left ventricular systolic function suggests simple pump failure is unlikely. A diagnosis of pulmonary arterial hypertension is not recommended on echocardiographic criteria alone because the estimate of pulmonary artery (PA) pressure is derived from the velocity of the tricuspid regurgitant jet and is liable to a considerable degree of operator dependence. While the normal spirometry and radiographic findings do not exclude asthma, the clinical history as revealed by a HARQ score three times the upper limit of normal suggests airway reflux. This was supported by the airway $\mathrm{pH}$ and barium studies. Cough, wheezing and shortness of breath could be ascribed to aspiration and in the absence of a gold standard test, guidelines recommend therapeutic trials. ${ }^{2}$ However, the patient's symptoms, particularly the prominent dyspnoea, seem disproportionate and further information should be sought in regard to the abnormal cardiovascular findings.

GE: A proton pump inhibitor (PPI) and promotility treatment with metoclopramide were commenced and the cough improved. By contrast, the exercise induced dyspnoea and orthopnoea progressed to the point where his capacity to perform activities of daily living was impaired. His chest X-ray now showed cardiomegaly, blunted costophrenic angles and bilateral lower zone infiltrates. A further echocardiogram revealed concentric left ventricular hypertrophy with a speckled appearance and 'stiff' relaxation of the myocardium. The right atrium was dilated with an estimated PA systolic pressure of $55 \mathrm{~mm} \mathrm{Hg}+$ right atrial pressure.

AHM: The marked improvement in cough with anti-reflux medication supports the diagnosis of airway reflux. However, cardiac dysfunction appears to underlie the dyspnoea. The echocardiographic findings are typical of a restrictive cardiomyopathy due to an infiltrative or fibrotic process such as amyloidosis, sarcoidosis, haemochromatosis or Loeffler's syndrome. Endomyocardial biopsy is indicated and treatment with diuretics should be initiated.

ALC: Left and right heart catheterisation was performed. There was poor left ventricular systolic function (ejection fraction 30\%). The pulmonary artery pressure was $48 / 21 \mathrm{~mm} \mathrm{Hg}$ with a mean pulmonary artery wedge pressure of $30 \mathrm{~mm} \mathrm{Hg}$. Ventricular diastolic pressures were raised and showed a dip-and-plateau pattern consistent with a restrictive cardiomyopathy. Endomyocardial biopsies from the right ventricular apex showed eosinophilic material with positive Congo red staining and apple green birefringence under polarised light consistent with amyloid deposition (figure 1A). There was no fibrin deposition, iron stains were negative and there was no evidence of fibrosis or myocarditis.

IM: These findings confirm the diagnosis of cardiac amyloidosis. The disease can then be further characterised by serum electrophoresis to identify the type of protein responsible. The most common is amyloid A type protein, an acute phase reactant produced in response to various chronic diseases. Less common types include $\beta-2$ microglobulin related amyloidosis in patients on dialysis, 

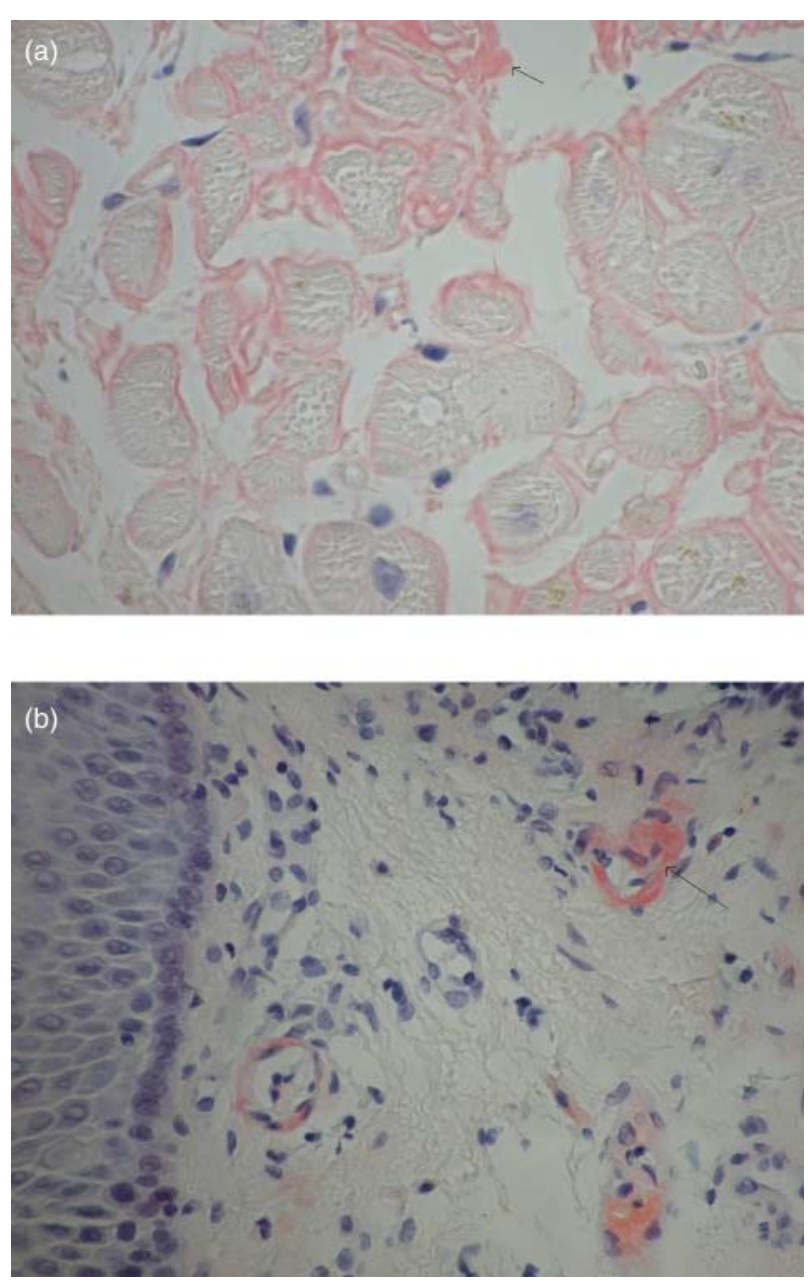

Figure 1 (A) Endomyocardial biopsy showing positive staining of the interstitium for amyloid (arrow). (Congo red $\times 400$ approx). (B) Oesophageal biopsy showing positive staining for amyloid in a small blood vessel in the lamina propria (arrow) (congo red $\times 200$ approx). This figure is only reproduced in colour in the online version.

which occurs as a result of failure to catabolise the protein in the kidney. There is also an inherited form due to mutations in the transthyretin gene (ATTR). Light chain (Primary, AL) amyloidosis is caused by deposition of immunoglobulin light chains or fragments, secondary to dysfunctional synthesis in monoclonal plasma cells. In this case, serum electrophoresis demonstrated lambda light chain excess and immunohistochemical analysis of the cardiac biopsies confirmed amyloid of the AL subtype. Multiple myeloma was excluded on bone marrow aspirate and skeletal survey. No amyloidogenic mutation was found on sequencing of the transthyretin gene.

GE: At review 8 months after presentation, the patient's symptoms had continued to deteriorate with some recurrence of cough, nausea, weight loss, peripheral oedema and a reduction in exercise tolerance to approximately $10 \mathrm{~m}$ walking. Upper gastrointestinal endoscopy was performed. Oesophageal biopsies showed eosinophilic material deposited in blood vessels which stained positively with Congo red and Sirius red (figure 1B).

IM: The diagnosis of amyloidosis involving both the heart and oesophagus is supported by the typical histology findings in biopsies from both locations. Cardiac involvement is common in light chain amyloid, typically presenting as a restrictive cardiomyopathy. The prognosis is poor. ${ }^{3}{ }^{4}$ Isolated pulmonary hypertension is rare in amyloidosis but can occur in older patients. $^{5-9}$ Amyloid deposition in the pulmonary vasculature has been proposed as a mechanism. Oesophageal amyloidosis has a variable presentation and may manifest as achalasia, oesophageal spasm, a non-specific motility disorder or decreased lower oesophageal sphincter pressure leading to reflux. ${ }^{10}$

GE: 10 months after the initial presentation the patient's cough and nausea had resolved with continuing PPI and motility therapy but there had been a worsening in breathlessness. He was using a wheelchair and had New York Heart Association class III dyspnoea. Referral was made to the National Amyloidosis Centre where cardiac transplant, low dose chemotherapy, and autologous stem cell transplantation were considered but the patient died 15 months after first developing symptoms and less than a year after presentation to secondary care.

AHM: This patient had primary (AL) amyloidosis presenting in an unusual manner with a chronic cough. The clinical characteristics such as the association with food and talking ${ }^{11}$ provided a strong clue to the aetiology which was confirmed by objective testing. Gastro-oesophageal complications of amyloidosis are common $^{12}$ and it is perhaps only with the growing realisation that cough is a frequent manifestation of gastrointestinal (GI) pathology that a link was made. The mechanism of oesophageal dysfunction is obscure since amyloid deposition was confined to the vessels and there was no primary oesophageal smooth muscle infiltration. Involvement of the vasa nervorum may have led to vagal autonomic dysfunction.

The key learning points from this case are:

1. Chronic cough is a common manifestation of upper GI pathology, revealed by its characteristic history.

2. Echocardiographic evidence of pulmonary hypertension requires careful interpretation and should not be the sole criterion for diagnosis.

3. Amyloidosis is rare and may be easily missed if apparently disparate symptoms are considered in isolation.

4. The incongruence of symptoms within an apparently established diagnosis should prompt reconsideration of the underlying mechanism of disease.

Acknowledgements The authors are grateful to Dr Anne Campbell, Consultant Histopathologist, Hull and East Yorkshire Hospitals for her assistance with the preparation of the figures.

Competing interests None.

Provenance and peer review Not commissioned; internally peer reviewed.

\section{REFERENCES}

1 Morice $A H$, Faruqi $S$, Wright $C E$, et al. The cough hypersensitivity syndrome: a distinct clinical entity. Lung 2011;189:73-9.

2 Morice AH, McGarvey L, Pavord I, on behalf of the British Thoracic Society Cough Guideline Group. Recommendations for the management of cough in adults. Thorax 2006;61:i1-24

3 Cacoub P, Axler O, De Zuttere D, et al. Amyloidosis and cardiac involvement. Ann Med Interne (Paris) 2000;151:611-17.

4 Michael M, Kastritis E, Delimpassi S, et al. Clinical characteristics and outcome of primary systemic light-chain amyloidosis in Greece. Clin Lymphoma Myeloma Leuk 2010;10:56-61.

5 Eder L, Zisman D, Wolf R, et al. Pulmonary hypertension and amyloidosis—an uncommon association: a case report and review of the literature. J Gen Intern Med 2007;22:416-19.

6 Lehtonen J, Kettunen P. Pulmonary hypertension as a dominant clinical picture in a case of amyloidosis and smoldering multiple myeloma. Int J Cardiol 2007;115: e29-30. 
7 Dingli D, Utz JP, Gertz MA. Pulmonary hypertension in patients with amyloidosis. Chest 2001;120:1735-8.

8 Chapman AD, Brown PA, Kerr KM. Right heart failure as the dominant clinical picture in a case of primary amyloidosis affecting the pulmonary vasculature. Scott Med J 1999:44:116-17.

9 Shiue ST, McNally DP. Pulmonary hypertension from prominent vascular involvement in diffuse amyloidosis. Arch Intern Med 1988;148:687-9.
10 Shatnawei A, Dasari V, Dumot J, et al. Two cases of gastric and esophageal amyloidosis. Gastroenterol Hepatol 2009;5:571-4.

11 Everett CF, Morice AH. Clinical history in gastroesophageal cough. Respir Med 2007;101:345-8.

12 James DG, Zuckerman GR, Sayuk GS, et al. Clinical recognition of Al type amyloidosis of the luminal gastrointestinal tract. Clin Gastroenterol Hepatol 2007:5:582-8. 